\title{
Complete pathological response (ypTONOMO) after preoperative chemotherapy alone for stage IV rectal cancer
}

Surennaidoo P Naiken ${ }^{1 *}$, Christian Toso ${ }^{1}$, Laura Rubbia-Brandt ${ }^{2}$, Theodoros Thomopoulos ${ }^{1}$, Arnaud Roth ${ }^{3}$, Gilles Mentha', Philippe Morel ${ }^{1}$ and Pascal Gervaz ${ }^{1}$

\begin{abstract}
Background: Complete pathological response occurs in 10-20\% of patients with rectal cancer who are treated with neoadjuvant chemoradiation therapy prior to pelvic surgery. The possibility that complete pathological response of rectal cancer can also occur with neoadjuvant chemotherapy alone (without radiation) is an intriguing hypothesis.

Case presentation: A 66-year old man presented an adenocarcinoma of the rectum with nine liver metastases (T3N1M1). He was included in a reverse treatment, aiming at first downsizing the liver metastases by chemotherapy, and subsequently performing the liver surgery prior to the rectum resection. The neoadjuvant chemotherapy consisted in a combination of oxaliplatin, 5-FU, irinotecan, leucovorin and bevacizumab (OCFL-B). After a right portal embolization, an extended right liver lobectomy was performed. On the final histopathological analysis, all lesions were fibrotic, devoid of any viable cancer cells. One month after liver surgery, the rectoscopic examination showed a near-total response of the primary rectal adenocarcinoma, which convinced the colorectal surgeon to perform the low anterior resection without preoperative radiation therapy. Macroscopically, a fibrous scar was observed at the level of the previously documented tumour, and the histological examination of the surgical specimen did not reveal any malignant cells in the rectal wall as well as in the mesorectum. All 15 resected lymph nodes were free of tumour, and the final tumour stage was ypTONOMO. Clinical outcome was excellent, and the patient is currently alive 5 years after the first surgery without evidence of recurrence.
\end{abstract}

Conclusion: The presented patient with stage IV rectal cancer and liver metastases was in a unique situation linked to its inclusion in a reversed treatment and the use of neoadjuvant chemotherapy alone. The observed achievement of a complete pathological response after chemotherapy should promote the design of prospective randomized studies to evaluate the benefits of chemotherapy alone in patients with stages II-III rectal adenocarcinoma (without metastasis).

Keywords: Pathological complete response, Stage iv rectal cancer, Preoperative chemotherapy, Oxaliplatin

\footnotetext{
* Correspondence: drnaikensuren@hotmail.com

${ }^{1}$ Services de chirurgie viscérale et transplantation, Département de chirurgie, Hôpitaux Universitaires de Genève, Rue Gabrielle-Perret-Gentil 4, Genève 14 1211, Suisse

Full list of author information is available at the end of the article
} 


\section{Background}

Colorectal cancer (CRC) is a major public health problem $[1,2]$. Over the last two decades, its management has improved significantly, in parallel to the implementation of dedicated multidisciplinary oncological rounds $[3,4]$. Routine staging is now in place in almost all centers with the use of MRI and/or endo-rectal ultrasonography. Surgery has been standardized thanks to the popularization of the total mesorectal excision technique. In addition, neoadjuvant treatment has been refined with the use of chemoradiotherapy (CRT) in selected patients with locally advanced cancer. More recently, the survival of patients with metastatic rectal cancer has also improved thanks to the availability of new potent drug combinations.

To illustrate these improvements in the (neo-) adjuvant treatments, up to $10 \%$ of patients with stage IV CRC do not have detectable liver metastasis anymore on pathology following chemotherapy [5,6]. Pathologic complete response (pCR) also occurs in 10-20\% of patients with rectal cancer who are treated with neoadjuvant chemo radiotherapy prior to pelvic surgery [7]. The current standard management of patients with rectal cancer reaching the peri-rectal fat (cT3-T4N0) and/or with a clinical evidence of lymphnode invasion (cTxN1) includes a neoadjuvant chemoradiotherapy [8]. At our institution, for patients with advanced synchronous colorectal liver metastasis (CRLM) and a non- obstructive primary tumor, a reversed protocol $[9,10]$ is recommended. They include patients with multiple, often large metastases not accessible for minor (up to two segments) up-front liver resection [10].
This strategy controls the CRLM and the primary tumour, hence increasing the curative liver metastatic resection. In our initial study [11], patients with advanced liver metastases (clinical risk score [CRS] of 3-5), were treated with highly effective chemotherapy, proving respectability and survival rate were better than expected. Neoadjuvant chemotherapy has helped achieving control of the CRLM in more than $80 \%$ of cases, and of the primary tumour (as well as of invaded lymph-nodes) in more than $60 \%$ of cases [12]. A total of 92 patients have been now managed by a reversed treatment, and seven patients demonstrated complete histological responses of all metastases after chemotherapy.

The possibility that $\mathrm{pCR}$ of rectal cancer may occur without neoadjuvant radiation therapy is an intriguing hypothesis, which could promote the use of neoadjuvant chemotherapy alone also for non-metastatic colorectal cancer. The described patient with rectal cancer and liver metastasis was included in a "reversed" management protocol, aiming at treating the disease by chemotherapy first, and further resecting the liver metastases and the primary rectal cancer sequentially [9-11]. This unique type of management allowed for the assessment of the pathological response to chemotherapy alone.

\section{Case presentation}

A 66-year old man presented with an adenocarcinoma of the rectum located $10 \mathrm{~cm}$ from the anal verge (uT3N1) (Figure 1). Thoraco-abdominal PET/CT scan revealed nine liver metastases (Figure 2). There were two lesions in

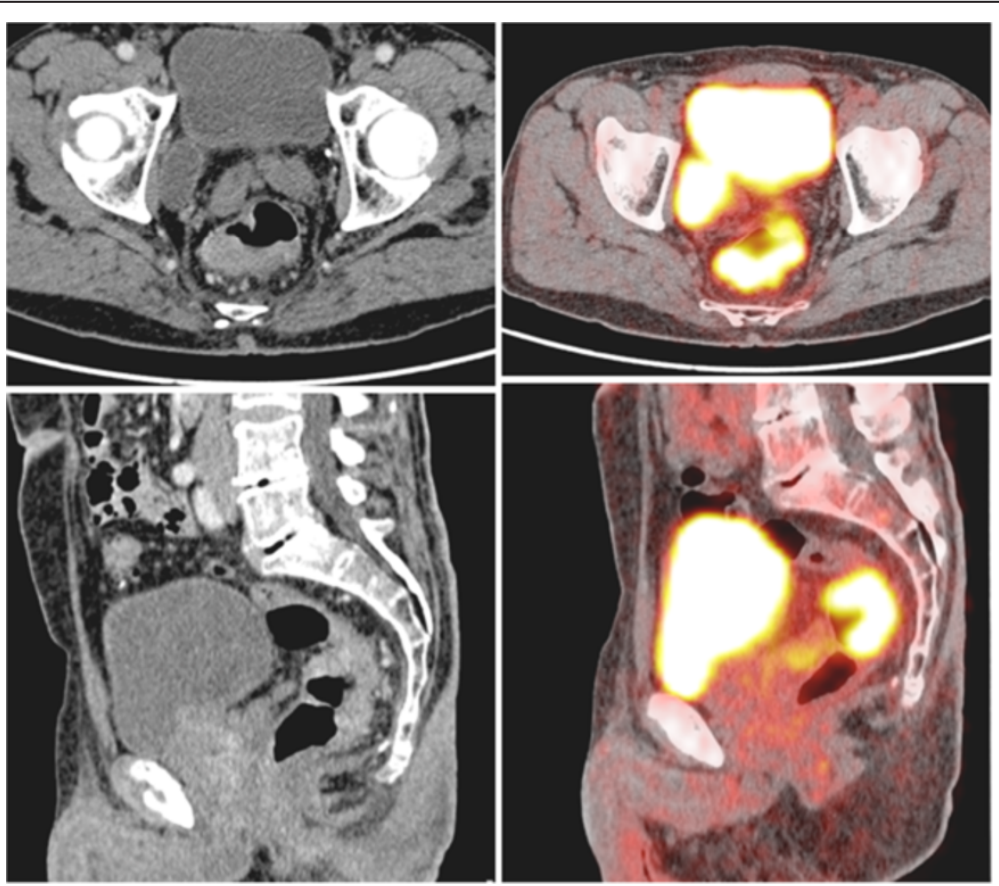

Figure 1 Radiological findings of the primary tumour. CT scan and Pet-scan of the rectal tumour prior to chemotherapy. 


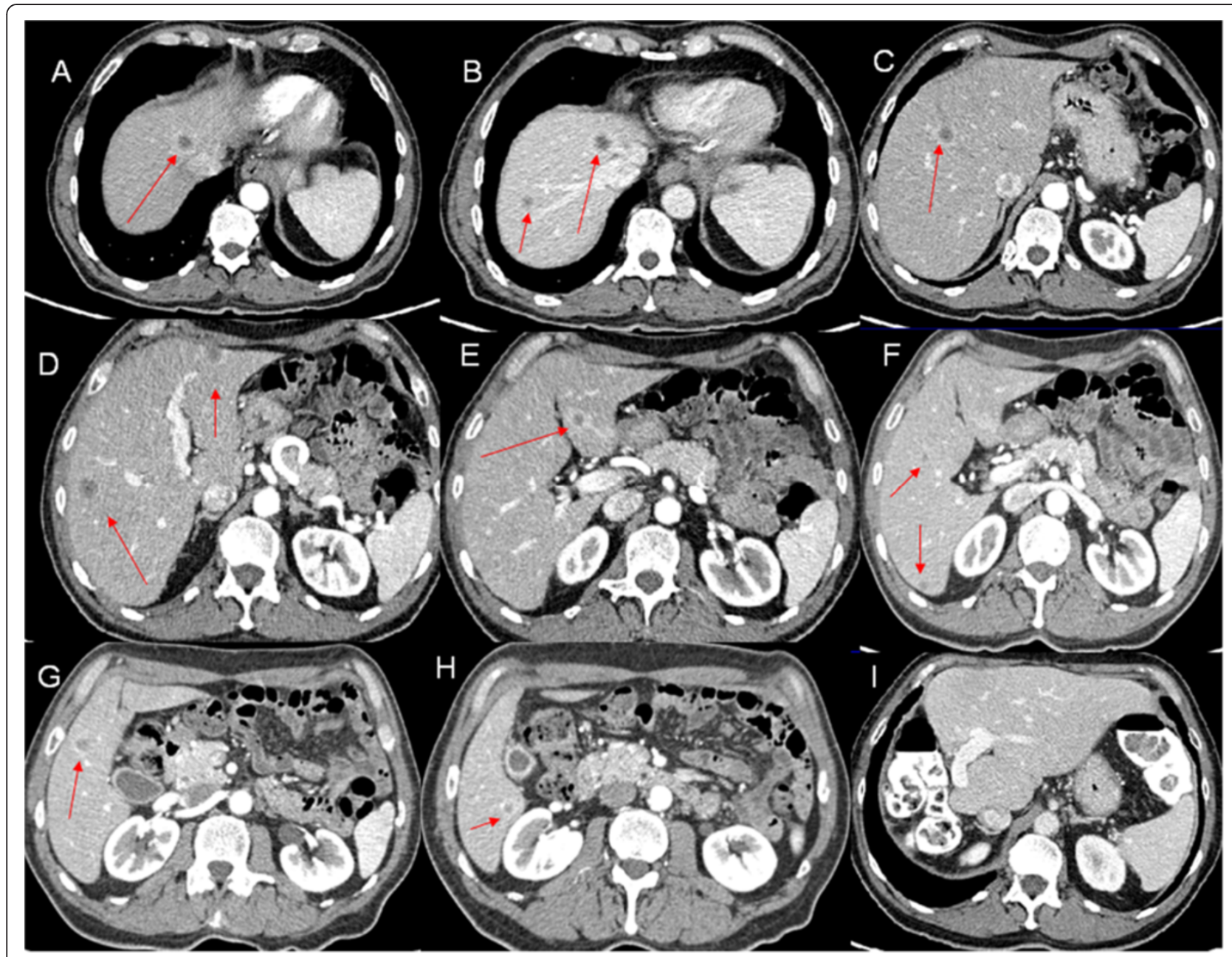

Figure 2 Computed tomography scan of liver metastases prior and after surgery: axial view A- H). Liver metastases in all liver segments except segment 1. I) CT scan after surgery. extended right hepatectomy showing no recurrence.

the segment III, three in segment IV, one between segment V and VII, and three in segment VI. The carcinoembryonic antigen (CEA) was $8.3 \mu \mathrm{G} / \mathrm{L}$. The clinical risk score was of two according to Fong's classification (positive lymph node status, and multiple synchronous liver metastases).

Neoadjuvant chemotherapy consisted in a combination of oxaliplatin, 5-fluorouracil, irinotecan, leucovorin and bevacizumab (OCFL-B). Our strategy aimed at controlling the metastatic disease and performing liver surgery prior to rectal surgery, according to a "reversed" protocol previously described [9,11]. After three cycles, the patient did not report rectal bleeding anymore, and a major response of the liver metastases was documented on magnetic resonance imaging (MRI); all nine secondary tumours had decreased in size. After a right portal vein embolization, an extended right liver lobectomy with the local resection of two lesions in segment III was performed. On the final histopathological analysis, all lesions were fibrotic nodules, devoid of any viable cancer cells and compatible with previous metastases.

One month after liver surgery, a rectoscopic examination showed a near-total response of the primary rectal adenocarcinoma, which convinced the colorectal surgeon to proceed with pelvic surgery without preoperative radiation therapy. A low anterior resection of the rectum was carried out. Macroscopically, a fibrous scar was observed at the level of the previously documented tumour, and the histological examination of the surgical specimen did not reveal any malignant cells in the rectal wall as well as in the mesorectum. All 15 removed lymph nodes were free of tumour, and the final tumour stage was therefore ypTONOM0 (Figure 3).

This response was associated to an excellent clinical outcome: although the patient initially presented with bilobar liver metastases, he is currently alive without signs of recurrence five years after surgery. This confirms the advantages of preoperative chemotherapy and resection 

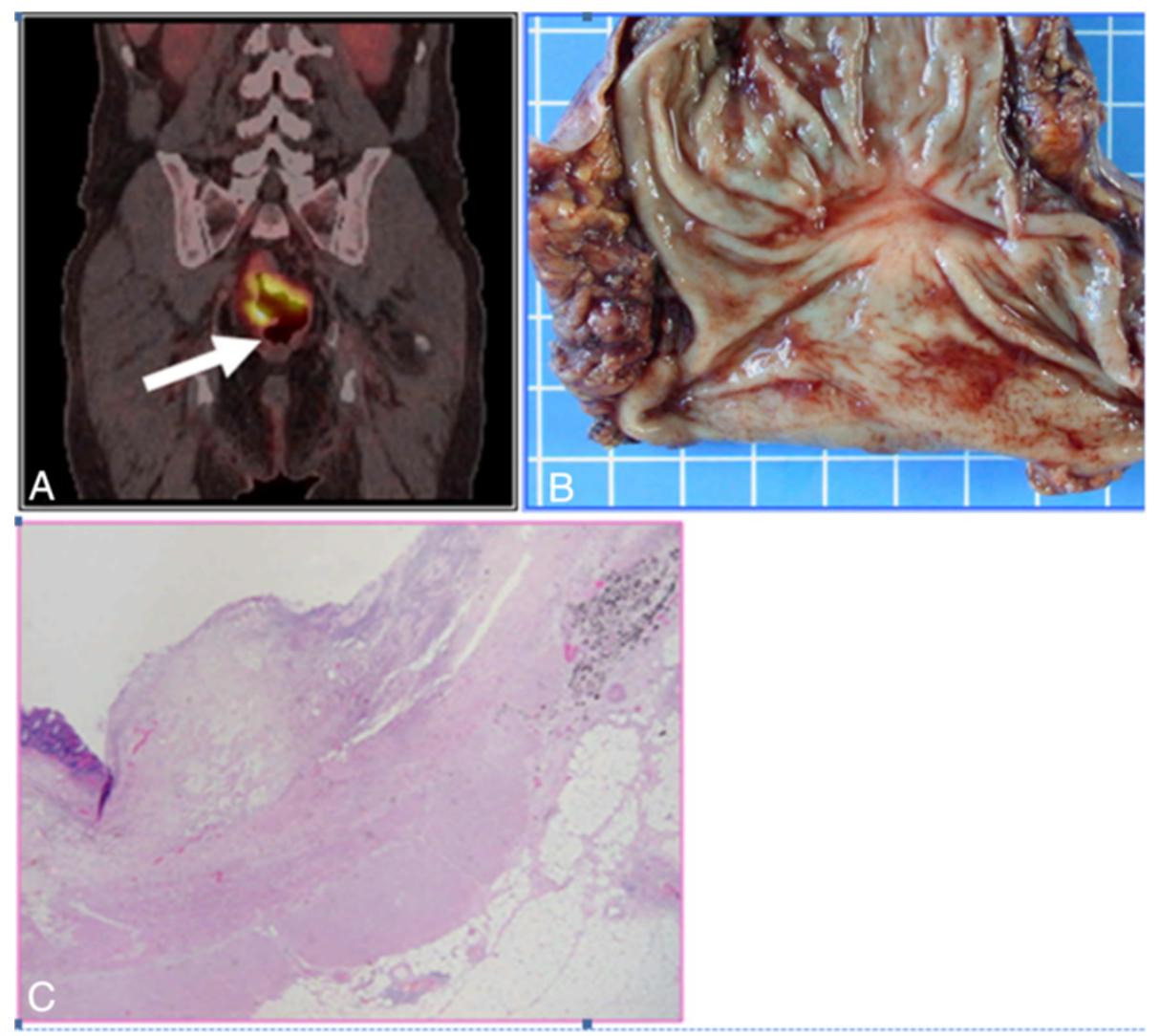

Figure 3 Radiological appearance, operative specimen and histological profile of the primary tumour. A. Pre-treatment PET scan showing hypermetabolic lesion of the mid rectum. B. Operative specimen of low anterior resection showing a fibrous scar in the mid rectum.

C. Histological analysis of the operative specimen, showing the absence of tumour cells and the presence of fibrous tissue within the partially reepithelialised rectal wall.

of colorectal liver metastasis to achieve pathologic response and hence predicts the survival rates [13].

\section{Conclusion}

The current standard of care of patients with locally advanced rectal cancer includes the use of neoadjuvant chemoradiotherapy. Overall, $10-20 \%$ of patients achieve a complete rectal pathological response after chemoradiation. Some investigators have even demonstrated a sustained clinical response in the absence of rectal resection, with a low recurrence rate of $4.6 \%$, and five-year overall and disease-free survival rates of 96 and $72 \%[14,15]$.

Despite these benefits, pelvic radiotherapy is linked to a number of limitations. It induces a peri-rectal inflammation, and the use of chemotherapy-only could make surgery easier, with higher chances of achieving a resection with appropriate margins by a minimally invasive approach. The use of radiotherapy in the management of rectal cancer is making another curative pelvic radiation impossible for potential prostatic, gynecological, bladder or anal cancer. In addition, it has long-term impact on anorectal, urinary and sexual function [16,17]. Finally, the recent improvements in the management of rectal cancer, including chemoradiotherapy and total mesorectal excision, have no impact on known or microscopic distant metastases.

The present patient with $\mathrm{pCR}$ received a chemotherapy combining oxaliplatin, 5-fluorouracil, irinotecan, leucovorin and bevacizumab. Oxaliplatin-based neoadjuvant chemotherapy, currently mainly used in stage IV patients, may represent an attractive alternative to the neoadjuvant radiation therapy for rectal cancer. Induction chemotherapy has the added advantage of earlier administration of systemic therapy and may improve the control or the prevention of distant metastasis.

Prospective randomized studies should be carried out to demonstrate effectiveness of systemic therapy into combined-modality programs and to evaluate the benefit of chemotherapy alone in selected patients with stages II-III rectal adenocarcinoma with or without distant metastasis. 


\section{Consent}

"Written informed consent was obtained from the patient for publication of this Case report and any accompanying images. A copy of the written consent is available for review by the Editor of this journal."

\section{Abbreviations}

CRC: Colorectal cancer; CRLM: Colorectal liver metastasis; 5-FU irinotecan: Oxaliplatin; OCFL-B: Leucovorin and bevacizumab; TME: Total mesorectal excision; CRT: Chemoradiotherapy; pCR: Pathologic complete response; CEA: Carcinoembryonic antigen; MRI: Magnetic resonance imaging.

\section{Competing interests}

The authors declare that they have no competing interests.

\section{Authors' contributions}

$L R B, A R, G M, P M, P G$ performed the clinical follow-up of the patient. GM, $P G$ performed the surgery. $C T, T, P G$ and $S N$ have made substantial contributions to conception and design, or acquisition of data, or analysis and interpretation of data. Furthermore, all authors have been involved in revising the manuscript critically for important intellectual content read and approved the final manuscript.

\section{Acknowledgement}

Christian Toso was supported by a Professorship from the Swiss National Science Foundation (PPOOP3_139021).

\section{Author details}

'Services de chirurgie viscérale et transplantation, Département de chirurgie, Hôpitaux Universitaires de Genève, Rue Gabrielle-Perret-Gentil 4, Genève 14 1211, Suisse. ${ }^{2}$ Services de pathologie clinique, Hôpitaux Universitaires de Genève, Rue Gabrielle-Perret-Gentil 4, Genève 14 1211, Suisse. ${ }^{3}$ Service d'oncochirurgie, Hôpitaux Universitaires de Genève, Rue

Gabrielle-Perret-Gentil 4, Genève 14 1211, Suisse.

Received: 25 March 2013 Accepted: 11 December 2013

Published: 17 January 2014

\section{References}

1. Ferlay J, Shin HR, Bray F, Forman D, Mathers C, Parkin DM: GLOBOCAN 2008 v2.0, Cancer Incidence and Mortality Worldwide: IARC CancerBase No. 10 [Internet]. Lyon, France: International Agency for Research on Cancer; 2010. Available from: http://globocan.iarc.fr.

2. Bray F, Ren JS, Masuyer E, Ferlay J: Estimates of global cancer prevalence for 27 sites in the adult population in 2008. Int J Cancer 2013, 132(5):1133-1145. doi: 10.1002/ijc.27711. Epub 2012 Jul 26.

3. Beart RW Jr: Multidisciplinary management of patients with advanced rectal cancer. Clin Cancer Res 2007, 13(22 Pt 2):6890s-6893s.

4. Cervantes A, Rodríguez-Braun E, Navarro S, Hernández A, Campos S, García-Granero E: Integrative decisions in rectal cancer. Ann Oncol 2007, 18(Suppl 9):127-131.

5. Rubbia-Brandt L, Giostra E, Brezault C, Roth AD, Andres A, Audard V, Sartoretti P, Dousset B, Majno PE, Soubrane O, Chaussade S, Mentha G, Terris B: Importance of histological tumor response assessment in predicting the outcome in patients with colorectal liver metastases treated with neo-adjuvant chemotherapy followed by liver surgery. Ann Oncol 2007, 18:299-304.

6. Adam R, Wicherts DA, de Haas RJ, Aloia T, Levi F, Paule B, Guettier C, et al: Complete pathologic response after preoperative chemotherapy; myth or reality? J Clin Oncol 2008, 26:1635-1641.

7. O'Neill BDP, Brown G, Heald RJ, Cunningham D, Tait DM: Non-operative treatment after neoadjuvant chemoradiotherapy for rectal cancer. Lancet Oncol 2007, 8:625-633.

8. Van Cutsem E, Dicato M, Haustermans K, Arber N, Bosset JF, Cunningham D, et al: The diagnosis and management of rectal cancer: expert discussion and recommendations derived from the 9th world congress on gastrointestinal cancer, Barcelona. Ann Oncol 2008 Jun, 19(Suppl 6):1-8. doi: 10.1093/annonc/mdn358. 2007.
9. Mentha G, Roth AD, Terraz S, Giostra E, Gervaz P, Andres A, Morel P, Rubbia-Brandt L: 'Liver first' approach in the treatment of colorectal cancer with synchronous liver metastases. Dig Surg 2008, 25:430-435.

10. Mentha G, Terraz S, Andres A, Toso C, Rubbia-Brandt L, Majno P: Operative management of colorectal liver metastases. Semin Liver Dis 2013, 33(3):262-272. doi: 10.1055/s-0033-1351785. Epub 2013 Aug 13.

11. Mentha G, Majno PE, Andres A, Rubbia-Brandt L, Morel P, Roth AD: Neoadjuvant chemotherapy and resection of advanced synchronous liver metastases before treatment of the colorectal primary. Br J Surg 2006, 93:872-878.

12. Gervaz P, Rubbia-Brandt L, Andres A, et al: Neoadjuvant chemotherapy in patients with stage IV colorectal cancer: a comparison of histological response in liver metastases, primary tumors, and regional lymph nodes. Ann Surg Oncol 2010, 17(10):2714-2719.

13. DG 3rd B, Kishi Y, Maru DM, Kopetz S, Chun YS, Overman MJ, Fogelman D, Eng C, Chang DZ, Wang H, Zorzi D, Ribero D, Ellis LM, Glover KY, Wolff RA, Curley SA, Abdalla EK, Vauthey JN: Pathologic response to preoperative chemotherapy: a new outcome end point after resection of hepatic colorectal metastases. Clin Oncol 2008, 26(33):5344-5351. doi: 10.1200/ JCO.2008.17.5299. Epub 2008 Oct 20.

14. Habr-Gama A, Perez RO, S̃ao Julĩao GP, Proscurshim I, Gama-Rodrigues J: Nonoperative approaches to rectal cancer: a critical evaluation. Semin Radiat Oncol 2011, 21:234-249.

15. Glynne-Jones R, Hughes R: Critical appraisal of the 'wait and see' approach in rectal cancer for clinical complete ressponders after chemoradiation. Br J Surg 2012, 99(7):897-909. doi: 10.1002/bjs.8732. Epub 2012 Apr 27.

16. Gervaz PA, Wexner SD, Pemberton JH: Pelvic radiation and anorectal function: introducing the concept of sphincter-preserving radiation therapy. J Am Coll Surg 2002, 195:387-394.

17. Bujko K, Nowacki MP, Nasierowska-Guttmejer A, Michalski W, Bebenek M, Kryj M: Long-term results of a randomized trial comparing preoperative short-course radiotherapy with preoperative conventionally fractionated chemoradiation for rectal cancer. Br J Surg 2006, 93:1215-1223.

doi:10.1186/1471-2482-14-4

Cite this article as: Naiken et al:: Complete pathological response (ypTONOMO) after preoperative chemotherapy alone for stage IV rectal cancer. BMC Surgery 2014 14:4.

\section{Submit your next manuscript to BioMed Central and take full advantage of:}

- Convenient online submission

- Thorough peer review

- No space constraints or color figure charges

- Immediate publication on acceptance

- Inclusion in PubMed, CAS, Scopus and Google Scholar

- Research which is freely available for redistribution

Submit your manuscript at www.biomedcentral.com/submit
C Biomed Central 